\title{
Genetic characterization of suspected MODY patients in Tunisia by targeted next-generation sequencing
}

\author{
Hamza Dallali ${ }^{1,2} \cdot$ Serena Pezzilli, ${ }^{3,4} \cdot$ Meriem Hechmi $^{1,2} \cdot$ Om Kalthoum Sallem ${ }^{5} \cdot$ Sahar Elouej $^{1,6} \cdot$ Haifa Jmel $^{1,7}$. \\ Yosra Ben Halima ${ }^{1,8}$. Mariem Chargui ${ }^{1}$ - Mariem Gharbi ${ }^{1}$ - Luana Mercuri ${ }^{3}$. Federica Alberico ${ }^{3}$. Tommaso Mazza ${ }^{9}$. \\ Afaf Bahlous $^{10}$. Melika Ben Ahmed ${ }^{11}$. Henda Jamoussi ${ }^{1,12}$. Abdelmajid Abid ${ }^{1,12}$. Vincenzo Trischitta ${ }^{3,4}$. \\ Sonia Abdelhak ${ }^{1,8} \cdot$ Sabrina Prudente ${ }^{3} \cdot$ Rym Kefi $^{1,8}$ (])
}

Received: 13 October 2018 / Accepted: 25 December 2018

(c) Springer-Verlag Italia S.r.l., part of Springer Nature 2019

\begin{abstract}
Aims Maturity Onset Diabetes of the Young (MODY) is a monogenic form of diabetes with autosomal dominant inheritance pattern. The diagnosis of MODY and its subtypes is based on genetic testing. Our aim was investigating MODY by means of next-generation sequencing in the Tunisian population.

Methods We performed a targeted sequencing of 27 genes known to cause monogenic diabetes in 11 phenotypically suspected Tunisian patients. We retained genetic variants passing filters of frequency in public databases as well as their probable effects on protein structures and functions evaluated by bioinformatics prediction tools.

Results Five heterozygous variants were found in four patients. They include two mutations in HNF1A and GCK that are the causative genes of the two most prevalent MODY subtypes described in the literature. Other possible mutations, including novel frameshift and splice-site variants were identified in $A B C C 8$ gene.

Conclusions Our study is the first to investigate the clinical application of targeted next-generation sequencing for the diagnosis of MODY in Africa. The combination of this approach with a filtering/prioritization strategy made a step towards the identification of MODY mutations in the Tunisian population.
\end{abstract}

Keywords MODY $\cdot$ Genetic testing $\cdot$ Next-generation sequencing $\cdot$ Targeted gene sequencing

\section{Introduction}

Maturity Onset Diabetes of the Young (MODY) is a monogenic form of non-insulin-dependent diabetes that classically presents in a lean individual with non ketotic hyperglycaemia and an autosomal dominant inheritance pattern. Most cases are diagnosed before the age of 25 [1].

MODY (OMIM Entry: \#606391) has been recognized as a clinically and genetically heterogeneous condition. To date, more than 1000 variants were identified in at least 14

Managed by Massimo Porta.

Electronic supplementary material The online version of this article (https://doi.org/10.1007/s00592-018-01283-5) contains supplementary material, which is available to authorized users.

Rym Kefi

Rym.Kefi@pasteur.tn; rym.kefi@ pasteur.utm.tn

Extended author information available on the last page of the article genes which define MODY subtypes [2-4]. Some of these genes are involved in pancreas and beta-cell development, while others play crucial roles in the various steps of insulin secretion, such as glucose sensing, and its metabolism in the beta cells [5].

Prevalence of MODY is difficult to estimate due to the overlapping of its clinical features with those of type 1 and type 2 diabetes, and therefore most cases are missed [6]. Some studies have reported that MODY accounts for 1-5\% of the young-onset diabetes population [7-10].

Classification of MODY subtypes is based on underlying the genetic variation on the candidate genes. The genes encoding the hepatic transcription factors HNF1A and $H N F 4 A$, along with the gene encoding the glucokinase, account for approximately $95 \%$ of all identified MODY cases [11].

Diagnosing MODY in young patients with diabetes is crucial to provide accurate counselling about the predicted clinical outcomes. Moreover, it allows subsequent 
identification of affected family members, and most importantly, appropriate management [12]. Indeed, misdiagnosis of MODY as type 1 diabetes (T1D) leads to inappropriate insulin therapy. For instance, in the case of HNF1A MODY, sulphonylureas, an inexpensive oral anti-diabetes medication, has shown its efficiency as a first-line treatment [13]. GCK MODY does not require medications as it leads to a mild fasting hyperglycaemia that is not associated with significant complications [14].

Traditional genetic testing of MODY consists of Sanger sequencing of the three most common MODY genes including 31 exons. However, this technique is expensive and time-consuming. The cost of sequencing the other candidate genes precludes further genetic testing, thus increasing the missing MODY cases [15].

The advent of high-throughput Next-Generation Sequencing (NGS) technologies has launched a revolution in screening strategies enabling simultaneous analysis of a panel of genes at a lower cost compared with testing few genes by Sanger sequencing. Indeed, NGS panels for MODY investigation studies were carried out in European, American and Asian populations [16-19].

In Tunisia, three studies screened for genetic variants in some known MODY genes by classical methods. The first study identified a variant in $H N F 4 A$ gene defining a MODY 1 subtype in only one patient, while 11 patients were found negative for variations in GCK, HNF1A, HNF4A, HNF1B, INS, IPFI and NEURODI genes [20]. Six among these 11 patients were also negative for variations in $P A X 4$ gene [21]. The last study identified only two GCK and HNF4A variants in three among 23 suspected Tunisian MODY patients [22]. These studies suggested that the most common genes known for MODY do not explain the majority of cases in Tunisians.

In this study, we investigate the genetic aetiology of 11 suspected Tunisian MODY patients by means of targeted NGS of a genes panel.

\section{Materials and methods}

\section{Study subjects}

This study was conducted according to the Declaration of Helsinki. It was approved by Pasteur Institute of Tunis ethics committee (Reference: 2016/19/I/LR11IPT05) and a written informed consent was obtained from the participants.

A total of 44 patients were recruited from the National Institute of Nutrition of Tunis based on the following criteria:

- Young onset of diabetes: age at diagnosis $<40$ years old.

- Positive family history of diabetes in at least two generations.

\section{- Absence of ketosis}

Autoantibodies to Glutamic Acid Decarboxylase (GAD), Islet Antigen 2 (IA2) as well as Islet Cell Antibodies (ICA) were analysed in the immunology laboratory of Institut Pasteur of Tunis as they are the biological markers of type 1 diabetes patients [23].

These criteria were used at the recruitment for primary classification aiming at excluding obvious type 1 and type 2 diabetes patients to increase the likelihood of MODY discovery.

Clinical characteristics, including demographic information, anthropometric measures, and diabetes history were collected during a survey prior to blood sample.

Metabolic parameters including fasting plasma glucose (FPG), glycated haemoglobin (HBA1c), cholesterol, highdensity lipoproteins (HDL), low-density lipoproteins (LDL), triglycerides (TG), creatinine and C-reactive protein (CRP) were measured in the biochemistry laboratory of Pasteur Institute of Tunis.

Among the 44 patients, almost full records were available for 11 who were selected for genetic testing with a gene panel-based next-generation sequencing approach. The other 33 patients were not included in the present study because of the lack of several additional clinical information (such as presence/absence of diabetes complications: micro/macro albuminuria, diabetic nephropathy, glycosuria, retinopathy as well as detailed treatment history) that does not allow us to comment on any genotype-phenotype correlation.

\section{Targeted next-generation sequencing}

Genomic DNA was isolated from the blood using the Flexigen DNA kit (QIAGEN). DNA quality was assessed using nanodrop spectrophotometer (Thermofisher scientific) and Qubit 3.0 (Life technologies).

Targeted capture and massive parallel sequencing of 27 genes reported to cause monogenic forms of diabetes were performed at CSS-Mendel Institute, Rome (Italy) as we recently described [24]. The list of genes is provided in Online Resource 1 (Table S1).

\section{Bioinformatics analysis}

The raw FASTQ files, generated by MiSeq reporter software were aligned to the hg 19 reference human genome. Then, variants were called for each individual sample in VCF files.

Coverage statistics were computed using Varaft 2.06 software [25]. Genetic variants were filtered to include only those with $>20 \times$ read depth and quality mapping score $\geq 30$.

Annotation of variants was carried out with Annovar database [26]. The likely pathogenicity of annotated genetic variants was evaluated according to the following pipeline: 
- Synonymous genetic variants were filtered out, keeping only nonsense, missense, frameshift insertions/deletions and splice-site variants, as they are more likely to have functional effect.

- Genetic variants with minor allele frequency (MAF) $>1 \%$ in gnomAD database (http://gnomad.broadinsti tute.org/) were excluded.

- The pathogenicity of the missense variants was assessed in silico by 13 prediction tools using a pathogenicity score as previously reported [24].

- The pathogenicity of splice-site variants was predicted in silico by the Human Splicing Finder [27] and SPiCE tools [28].

We searched bioinformatic databases: Pubmed (https:// www.ncbi.nlm.nih.gov/pubmed), Clinvar (https://www.ncbi. nlm.nih.gov/clinvar) and LOVD [29] for genetic variants surviving the filtering/prioritization pipeline to determine if they were previously reported and if so, their relevant studies and ontologies.

The following genetic variants were retained:

- Missense variants with a total predicted pathogenicity score $>7$.

- Nonsense and frameshift variations being by their intrinsic nature considered as pathogenic or likely pathogenic.

- Variants reported to alter splice sites by the Human Splicing Finder and SPiCE tools.

The retained variants were finally checked using the Mutalyzer program [30].

\section{Sanger sequencing}

Sanger sequencing was performed to confirm the likely pathogenic predicted variants in the probands, and to check the familial segregation when possible. In brief, genetic variants containing exons were amplified from DNA samples by polymerase chain reaction (PCR) using oligonucleotide primers designed by Primer3 software (available upon request). The resulting amplicons were sequenced on automated ABI3500 (Applied Biosystems, CA, USA) using the ABI prism Big Dye terminator v3.1 cycle sequencing kit. Sequence analysis was performed using BioEdit software [31].

\section{Results}

The clinical characteristics of the 11 Tunisian study participants are summarized in Table 1.

The exons of 27 genes were enriched with TruSeq Custom amplicon low-input library preparation kit and were sequenced with Illumina MiSeq technology. The average read depth across the targeted regions was $1422 \times$ with $94.2 \%$ covered $>20 \times$.

The filtering/prioritization pipeline resulted in the identification of three non-synonymous variants, one frameshift deletion and one splice-site variant in four patients (Fig. 1). They include two novel and three previously reported mutations (Table 2). Binary bioinformatic pathogenicity scores are provided in Online Resource 1 (Table S2).

The two novel variants were identified in the $A B C C 8$ gene and were carried by the patient MODY-TUN-P01. The first is a frameshift deletion resulting in the loss of approximately $50 \%$ of the amino acids of the $A B C C 8$ protein: c.2376delC/p.Phe793Serfs*71. The second is a splicesite variant: c. $4608+4 \mathrm{~A}>\mathrm{G}$, which alters the donor splice site near the exon 38 according to the Human Splice Finder and SPiCE tools. The patient developed a relatively late clinical manifestation of diabetes (at age of 32 years old) with a dramatic increase in glycaemia $(\mathrm{FPG}=22.7 \mathrm{mmol} / \mathrm{l}$ and $\mathrm{HBA} 1 \mathrm{c}>7 \%$ ) and a persistent polyuria and polydipsia. There are no signs of ketosis or positive pancreatic autoantibodies. Two months of insulin therapy resulted in FPG decline to $7.06 \mathrm{mmol} / \mathrm{l}$. Sanger sequencing confirmed the presence of the two heterozygous variants in the proband, the presence of only the frameshift deletion in the father having high TG, and the splice-site variant in the mother with type 2 diabetes mellitus.

The patient MODY-TUN-P15 carried a heterozygous missense variant in the $A B C C 8$ gene: c. $4606 \mathrm{G}>\mathrm{A} / \mathrm{p}$.Ala$1536 \mathrm{Thr}$. This genetic variant was predicted as pathogenic by 10 prediction tools and it is very rare according to gnomAD $\left(\mathrm{MAF}=8.12 \times 10^{-6}\right)$. It has an identifier in dbsnp database but without any clinical information. This patient has been diagnosed with fasting hyperglycaemia $(8.25 \mathrm{mmol} / \mathrm{l})$ at 10 years old. He had strong diabetes family history and has taken an oral anti-diabetes drug (OAD) for 25 years. The clinical follow-up demonstrated a progressive glycosuria, and the biochemical analysis upon enrolment revealed high TG and cholesterol levels as well as a persistent fasting hyperglycaemia. Pancreatic antibodies were negative and endogenous insulin secretion was suggested by a normal C-peptide level $(1.4 \mathrm{ng} / \mathrm{ml})$. Sanger sequencing confirmed the existence of the variant in the patient, but no family members were available to check its familial segregation.

In patient MODY-TUN-P12, we identified a missense variant in HNF1A gene: c.710A > G/p.Asn237Ser, that was attributed a pathogenic effect by 12 prediction tools. The patient was diagnosed with fasting hyperglycaemia $(7.38 \mathrm{mmol} / \mathrm{l})$, polyuria and polydipsia at 11 years old. He took both metformin and sulfonylurea, resulting in a decrease of FPG that has been then not stabilized within the range of 7-13 mmol/l due to an irregular uptake of the OAD according to the clinical follow-up. This patient has no signs of pancreatic antibodies. Biochemical analyses showed 


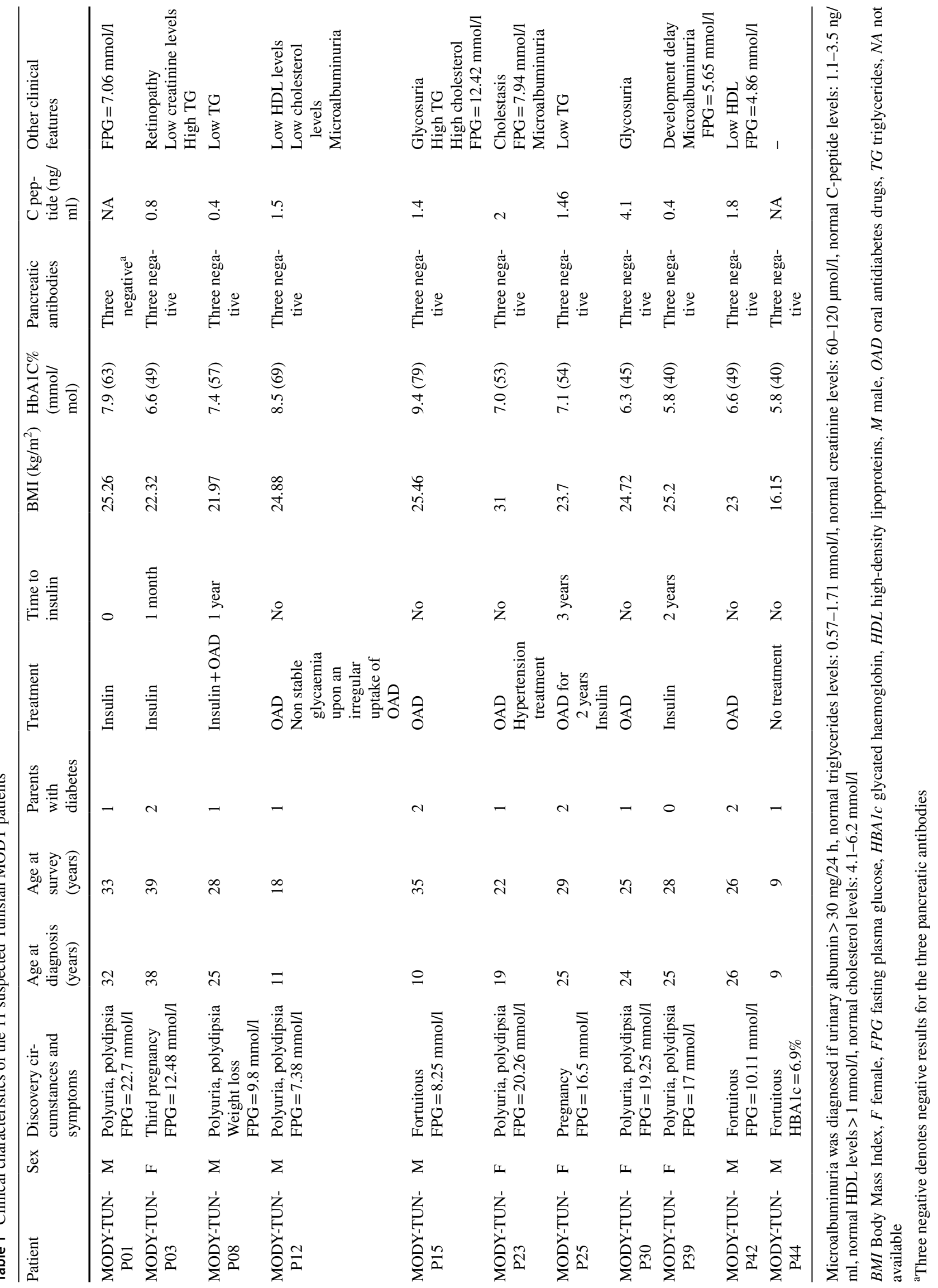



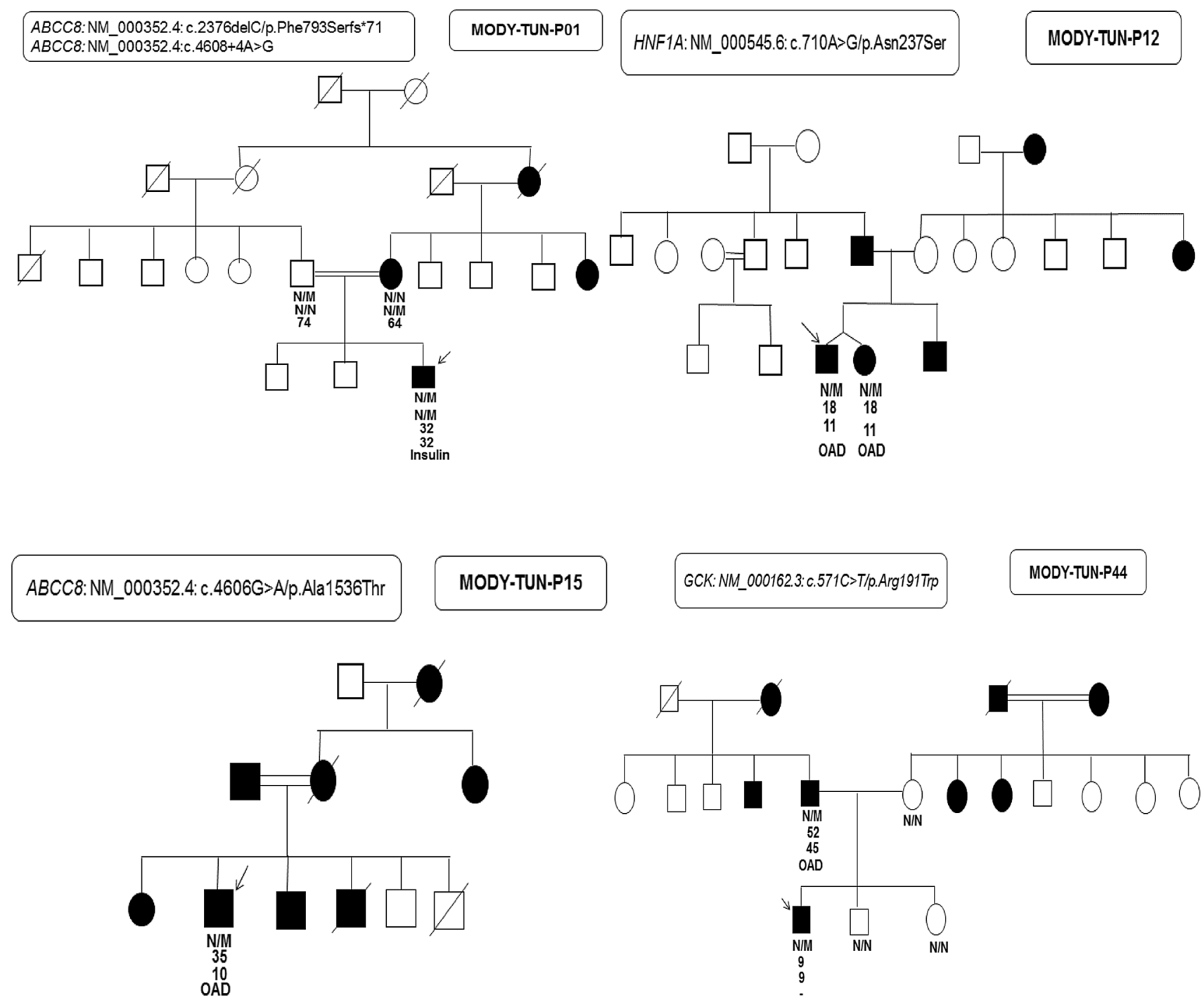

Fig. 1 Pedigrees of the four patient's families carrying the filtered genetic variants. The arrow indicates the proband, double lines indicate consanguineous matings, white squares and circles indicate healthy males and females, respectively, black squares and circles

indicate males and females with diabetes. N/M denotes mutation, while $\mathrm{N} / \mathrm{N}$ denotes no mutation. Below the genotype are: age at recruitment, age at diabetes diagnosis and specific anti-hyperglycaemia treatment

Table 2 List of the filtered genetic variants identified in the suspected Tunisian MODY patients

\begin{tabular}{|c|c|c|c|c|c|c|c|c|}
\hline Patient & Gene & Refseq & Genetic variant & Consequence & dbSNP & $\begin{array}{l}\text { gnomAD Fre- } \\
\text { quency }\end{array}$ & $\begin{array}{l}\text { Patho- } \\
\text { genicity } \\
\text { score }\end{array}$ & Reference \\
\hline \multirow[t]{2}{*}{ MODY-TUN-P01 } & $A B C C 8$ & NM_000352.4 & c.2376delC & p.Phe793Serfs*71 & - & - & - & Present study \\
\hline & & & c. $4608+4 \mathrm{~A}>\mathrm{G}$ & $\begin{array}{l}\text { Splice-site substi- } \\
\text { tution }\end{array}$ & - & - & - & Present study \\
\hline MODY-TUN-P12 & HNF1A & NM_000545.6 & c. $710 \mathrm{~A}>\mathrm{G}$ & p.Asn237Ser & - & - & 12 & LOVD [29] \\
\hline MODY-TUN-P15 & $A B C C 8$ & NM_000352.4 & c. $4606 \mathrm{G}>\mathrm{A}$ & p.Ala1536Thr & rs772332005 & $8.12 \mathrm{e}^{-06}$ & 10 & gnomAD \\
\hline MODY-TUN-P44 & $G C K$ & NM_000162.3 & c. $571 \mathrm{C}>\mathrm{T}$ & p.Arg $191 \operatorname{Trp}$ & - & $8.13 \mathrm{e}^{-06}$ & 12 & LOVD [29] \\
\hline
\end{tabular}

gnom $A D$ the genome aggregation database (http://gnomad.broadinstitute.org/), $d b S N P$ single nucleotide polymorphism database 
C-peptide value in the reference range $(1.5 \mathrm{ng} / \mathrm{ml})$. Sanger sequencing revealed the presence of the heterozygous genetic variant in both the proband and his twin sister who developed diabetes at approximately the same age. Parental samples were not available to check familial segregation.

The patient MODY-TUN-P44 was diagnosed with mild hyperglycaemia at the age of 9 . NGS results analysis showed that he carried a missense variant in $G C K$ gene: c. $571 \mathrm{C}>\mathrm{T} / \mathrm{p}$.Arg $191 \mathrm{Trp}$. This genetic variant was predicted as pathogenic by 12 bioinformatic tools and it is very rare according to gnomAD $\left(\mathrm{MAF}=8.13 \times 10^{-6}\right)$. This patient is a member of a family with diabetes history across three generations in both sides. Sanger sequencing revealed the presence of the genetic variant in the proband and only his father having type 2 diabetes mellitus, which points out an autosomal dominant inheritance pattern.

\section{Discussion}

In the current study, we performed the targeted sequencing of 27 genes reported to cause monogenic diabetes in suspected Tunisian MODY patients in order to detect the causative genetic variants. To our knowledge, this study is the first of this type to investigate the clinical application of NGS in the diagnosis of MODY in Africa.

The high throughput of sequencing technologies results in increased amount of genetic variants whose causative role in the investigated disease is questionable [32]. To surpass this problem, the likely pathogenicity of the observed genetic variants was evaluated by a combined filtering/prioritization strategy according to reads depth and quality, allele frequency, predicted functional impact by bioinformatic prediction tools known for their high classification records [24, 33], clinical features of patients as well as with extensive literature search for reported clinical and functional studies.

We identified 5 likely pathogenic mutations in 4 among 11 patients. They include two variants in the genes defining the two most prevalent MODY subtypes, which are HNF1A and $G C K$ accounting for roughly $70 \%$ of cases [34].

Variants in HNF1A gene are the most common causes of MODY, responsible for $52 \%$ of monogenic diabetes in the United Kingdom [6]. Moreover, they demonstrate high penetrance, in which almost $63 \%$ of mutation carriers develop diabetes before 25 years old [35]. Our assay results analysis revealed that both MODY-TUN-P12 and his twin sister carried a missense variant located in the highly conserved $\mathrm{POU}_{\mathrm{H}}$ domain of HNF1A, which initiates the interaction of the transcription factor protein with target genes expressed in the pancreas and the liver, and involved in glucose transport and metabolism. This variant was previously found in two suspected MODY females from England aged 12 and 15, respectively, by K. Colclough in Exeter hospital (United
Kingdom) [29]. All these arguments may suggest the possible causative effect of the identified variant within the $H N F 1 A$ gene.

The $G C K$ variant was identified in a 9-year-old boy diagnosed with slightly elevated HBA1c. According to LOVD database, it was previously reported in seven persons with diabetes of Brazilian and Italian ethnic origin with probable pathogenic effect towards MODY subtype [29]. The affected amino acid is localized in a functional domain that is crucial in the conformational change of the glucokinase to its active state for the catalysis of the glucose phosphorylation reaction [36].

The other three retained variants in our study were located in the $A B C C 8$ gene which encodes the sulphonyluria receptor 1 (SUR1) subunits of the ATP-sensitive potassium channel in the pancreatic beta cells. This channel links the insulin secretion by regulating the potassium ion flow across the beta cell membrane. The first is a novel splice-site variant in $A B C C 8$ gene: c. $4608+4 \mathrm{~A}>\mathrm{G}$, which was identified in the patient MODY-TUN-P01 as well as in his diabetic mother. Both "Human Splicing Finder" and "SPiCE" tools predicted splicing alteration through the possible break of the donor splice site located at $5^{\prime}$ of intron 38 . Therefore, the A to $G$ transition at four bp positions downstream of exon 38 may decrease the recognition of the intron 38 wild type donor site, which may lead to the activation of a cryptic splice site. We have analysed the $\pm 100 \mathrm{bp}$ region on each side of the exon-intron boundary using "Human Splicing Finder" to check the presence of potential splice sites. This shows the possibility of activation of a cryptic splice site at position 52 in the exon 38, which causes the loss of $12 \mathrm{bp}$ of this exon. Another possibility is the activation of a cryptic splice site in intron 38 at position c. $4608+21$, which may insert $20 \mathrm{bp}$ of intronic sequence between exons 38 and 39 . The aberrant splicing is predicted to result in a truncated protein due to a premature termination at codon 1550 . This would result in the obtaining of an $A B C C 8$ protein with 1549 among the original 1581 amino acids, including 13 different ones. Having said all this, we do acknowledge that functional experiments are needed to validate the real effect of this variant on the messenger RNA of $A B C C 8$ gene and to eventually address its role in concurring to cause hyperglycaemia.

In addition, this patient carried a novel frameshift deletion, c.2376delC/p.Phe793Serfs*71, most probably resulting in a loss of function of the $A B C C 8$ protein. This variant was also present in the non-diabetic father, which may rule out a probable effect on hyperglycaemia. Taken all together, while waiting for further and deeper studies, no firm conclusions can be drawn on the role of the two above-mentioned $A B C C 8$ variants on hyperglycaemia.

The third $A B C C 8$ variant occurred in the patient MODYTUN-P15 diagnosed with diabetes for 25 years since 10 years old, and who has a brother who died at 1 year old 
with neonatal hyperglycaemia. Missense variants in this gene were implicated in variable clinical phenotypes with glucose intolerance, mild hyperglycaemia or insulin-requiring diabetes from a young age (neonatal diabetes due to recessive mutations) to adulthood (MODY due to heterozygote mutations) even within the same pedigree [37-40].

Our study contributed to better understand the pathophysiology of diabetes in patients with genetic characterisation. In fact, the identification of the HNFIA mutation confirmed the sulphonylurea treatment for the patient MODY-TUNP12, who had unstable glycaemia upon an irregular uptake of his medications. For the patient MODY-TUN-P15, OAD treatment as a monotherapy provided good glycaemia control over approximately 25 years, which demonstrated the good response to OAD for this $A B C C 8$-MODY patient. However, the recent combination of hyperglycaemia, high TG and cholesterol levels with glycosuria should be noted as indicators towards the addition of insulin therapy. Indeed, $A B C C 8$ mutations were described in cases who successfully switched from insulin to sulfonylurea, as well as in insulin-requiring diabetic patients [38, 39]. Regarding the patient MODY-TUN-P01, he was maintained under insulin treatment, as deeper studies are required for the validation of the effect of $A B C C 8$ genetic variant. Finally, for the patient MODY-TUN-P44, recently diagnosed with diabetes, the identification of the causative mutation in $G C K$ gene denoted a MODY 2 subtype, which requires only a diet for a better management of the hyperglycaemia. Non-carrying mutation family members have benefited from a genetic counselling to ensure them that they did not have the risk factor for hyperglycaemia.

Interestingly, there is an early onset of retinopathy or microalbuminuria in 4 of the 11 suspected MODY patients. This observation can be explained for the patients MODYTUN-003, MODY-TUN-023 and MODY-TUN-039 by the late diagnosis of diabetes. For the patient MODY-TUN-P12, the irregular treatment with OAD led to hyperglycaemia during 7 years. This may cause vascular complications, which are known to be associated with significantly higher rates of microalbuminuria [41].

Genetic cause was not identified in 7 of the 11 selected patients. This result can be due to a certain clinical overlap between MODY and other forms of diabetes in these patients. Another explanation is that the cause of diabetes may be in currently unknown MODY genes. In addition, we do acknowledge that the exclusion of patients with hyperketonaemia, a clinical characteristic of T1D, might have excluded patients with MODY 3 who quite rarely may present as such condition [42, 43].

Targeted NGS assays to identify mutations causing MODY have been developed in previous studies using various capture kits. The first study, carried out by Ellard et al. on 2013, investigated 33 suspected MODY patients by massive parallel sequencing of 29 genes implicated in neonatal diabetes, MODY, maternally inherited diabetes and deafness (MIDD) or familial partial lipodystrophy [16]. Mutations were identified in five patients, including two mutations in the m.3243 region of the mitochondrial genome known to cause MIDD. This result denotes the overlapping criteria for the distinction of MODY from other forms of diabetes.

This first assay was followed by some studies testing for monogenic diabetes including MODY [17, 19, 24, 44, 45]. Recruitment criteria were relatively specific in each study. For example, the age at diagnosis threshold varied between 30 and 45 years in studies realized in Poland and Asian populations, respectively [44, 45]. Aranburu et al. investigated suspected American MODY patients with either linear history of diabetes or the absence of the type 1 diabetes-related pancreatic autoantibodies [19]. All these parameters were included in our recruitment strategy to evaluate the combination between clinical investigation and targeted NGS in the identification of MODY cases.

\section{Conclusion}

The present study reported the results of genetic investigation of Tunisian suspected MODY patients. This population is characterized by a particular structure, i.e. a very diverse genetic background and a high rate of consanguinity that influence its genetic make-up. Consequently, genes responsible for MODY might be different from those reported in other Caucasian populations. Indeed, our study shows that the most frequently reported genes are not apparently the major genes involved in MODY in our population. In this context, exome sequencing presents an alternative to look for genetic variants in not currently reported genes.

Acknowledgements We thank the patients, their parents and healthcare professionals who participated in this study. We also thank the CSSMendel Institute (Rome, Italy) for the collaboration and the provision of infrastructure for this research. This work was supported by Institut Pasteur of Tunis (PCI-15) and the Tunisian Ministry of higher Education and Scientific Research (LR11 IPT05). This study was partly supported by the Italian Ministry of Health ("Ricerca Corrente 20152017" to S. Prudente).

\section{Compliance with ethical standards}

Conflict of interest The authors declare that they have no conflict of interest.

Ethical approval All procedures performed in studies involving human participants were approved by Institut Pasteur of Tunis ethics committee (Reference: $2016 / 19 /$ I/LR11IPT05) and were in accordance with the 1964 Helsinki Declaration and its later amendments or comparable ethical standards. 
Informed consent Informed consent was obtained from all individual participants included in the study.

\section{References}

1. Hattersley A, Bruining J, Shield J et al (2009) The diagnosis and management of monogenic diabetes in children and adolescents. Pediatr Diabetes 10:33-42. https://doi.org/10.111 1/j.1399-5448.2009.00571.x

2. Kim SH (2015) Maturity-onset diabetes of the young: what do clinicians need to know? Diabetes Metab J 39:468-477. https:// doi.org/10.4093/dmj.2015.39.6.468

3. Prudente S, Jungtrakoon P, Marucci A et al (2015) Loss-of-function mutations in APPL1 in familial diabetes mellitus. Am J Hum Genet 97:177-185. https://doi.org/10.1016/j.ajhg.2015.05.011

4. Anik A, Çatli G, Abaci A, Böber E (2015) Maturity-onset diabetes of the young (MODY): an update. J Pediatr Endocrinol Metab 28:251-263. https://doi.org/10.1515/jpem-2014-0384

5. Timsit J, Saint-Martin C, Dubois-Laforgue D, Bellanné-Chantelot C (2016) Searching for maturity-onset diabetes of the young (MODY): when and what for? Can J Diabetes 40:455-461. https ://doi.org/10.1016/j.jcjd.2015.12.005

6. Shields BM, Hicks S, Shepherd MH et al (2010) Maturity-onset diabetes of the young (MODY): How many cases are we missing? Diabetologia 53:2504-2508. https://doi.org/10.1007/s0012 5-010-1799-4

7. Fendler W, Borowiec M, Baranowska-Jazwiecka A et al (2012) Prevalence of monogenic diabetes amongst Polish children after a nationwide genetic screening campaign. Diabetologia 55:26312635. https://doi.org/10.1007/s00125-012-2621-2

8. Irgens HU, Molnes J, Johansson BB et al (2013) Prevalence of monogenic diabetes in the population-based Norwegian childhood diabetes registry. Diabetologia 56:1512-1519. https://doi. org/10.1007/s00125-013-2916-y

9. Pihoker C, Gilliam LK, Ellard S et al (2013) Prevalence, characteristics and clinical diagnosis of maturity onset diabetes of the young due to mutations in HNF1A, HNF4A, and glucokinase: results from the SEARCH for diabetes in Youth. J Clin Endocrinol Metab 98:4055-4062. https://doi.org/10.1210/jc.2013-1279

10. Thanabalasingham G, Pal A, Selwood MP et al (2012) Systematic assessment of etiology in adults with a clinical diagnosis of young-onset type 2 diabetes is a successful strategy for identifying maturity-onset diabetes of the young. Diabetes Care 35:12061212. https://doi.org/10.2337/dc11-1243

11. Rubio-Cabezas O, Hattersley AT, Njølstad PR et al (2014) The diagnosis and management of monogenic diabetes in children and adolescents. Pediatr Diabetes 15:47-64. https://doi.org/10.1111/ pedi. 12192

12. Thanabalasingham G, Owen KR (2011) Diagnosis and management of maturity onset diabetes of the young (MODY). Bmj 343:d6044-d6044. https://doi.org/10.1136/bmj.d6044

13. Pearson ER, Starkey BJ, Powell RJ et al (2003) Mechanisms of disease genetic cause of hyperglycaemia and response to treatment in diabetes GLOSSARY. Lancet 362:1275-1281. https:// doi.org/10.1016/S0140-6736(03)14571-0

14. Stride A, Shields B, Gill-carey O et al (2014) Cross-sectional and longitudinal studies suggest pharmacological treatment used in patients with glucokinase mutations does not alter glycaemia. Diabetologia 57:54-56. https://doi.org/10.1007/s00125-013-3075-x

15. Johansson S, Irgens $\mathrm{H}$, Chudasama KK et al (2012) Exome sequencing and genetic testing for MODY. PLoS One 7:1-8. https ://doi.org/10.1371/journal.pone.0038050
16. Ellard S, Lango Allen H, De Franco E et al (2013) Improved genetic testing for monogenic diabetes using targeted nextgeneration sequencing. Diabetologia 56:1958-1963. https://doi. org/10.1007/s00125-013-2962-5

17. Bonnefond A, Philippe J, Durand E et al (2014) Highly sensitive diagnosis of 43 monogenic forms of diabetes or obesity through one-step pcrbased enrichment in combination with nextgeneration sequencing. Diabetes Care 37:460-467. https://doi. org/10.2337/dc13-0698

18. Gao R, Liu Y, Gjesing AP et al (2014) Evaluation of a target region capture sequencing platform using monogenic diabetes as a study-model. BMC Genet 15:6-8. https://doi. org/10.1186/1471-2156-15-13

19. Alkorta-Aranburu G, Carmody D, Cheng YW et al (2014) Phenotypic heterogeneity in monogenic diabetes: the clinical and diagnostic utility of a gene panel-based next-generation sequencing approach. Mol Genet Metab 113:315-320. https://doi. org/10.1016/j.ymgme.2014.09.007

20. Amara A, Chadli-Chaieb M, Ghezaiel H et al (2012) Familial early-onset diabetes is not a typical MODY in several Tunisian patients. Tunis Med 90:882-887

21. Amara A, Chadli-chaieb M, Chaieb L et al (2014) Challenges for molecular diagnosis of familial early-onset diabetes in unexplored populations. Iran J Public Health 43:1011-1013

22. Khelifa SB, Martinez R, Dandana A et al (2018) Maturity onset diabetes of the young (MODY) in Tunisia: low frequencies of GCK and HNF1A mutations. Gene 651:44-48. https://doi. org/10.1016/j.gene.2018.01.081

23. Mcdonald TJ, Colclough K, Brown R et al (2011) Islet autoantibodies can discriminate maturity-onset diabetes of the young (MODY) from Type1 diabetes. Diabet Med 28:1028-1033. https ://doi.org/10.1111/j.1464-5491.2011.03287.x

24. Pezzilli S, Ludovico O, Biagini T et al (2018) Insights from molecular characterization of adult patients of families with multigenerational diabetes. Diabetes 67:137-145. https://doi. org/10.2337/db17-0867

25. Desvignes J-P, Bartoli M, Delague V et al (2018) VarAFT: a variant annotation and filtration system for human next generation sequencing data. Nucleic Acids Res 46:W545-W553. https://doi. org/10.1093/nar/gky471

26. Wang K, Li M, Hakonarson H (2010) ANNOVAR: functional annotation of genetic variants from high-throughput sequencing data. Nucleic Acids Res 38:e164-e164. https://doi.org/10.1093/ nar/gkq603

27. Desmet FO, Hamroun D, Lalande M et al (2009) Human splicing finder: an online bioinformatics tool to predict splicing signals. Nucleic Acids Res 37:1-14. https://doi.org/10.1093/nar/gkp215

28. Leman R, Gaildrat P, Gac GL et al (2018) Novel diagnostic tool for prediction of variant spliceogenicity derived from a set of 395 combined in silico/in vitro studies: an international collaborative effort. Nucleic Acids Res 46:7913-7923. https://doi.org/10.1093/ nar/gky372

29. Fokkema IFAC, Taschner PEM, Schaafsma GCP et al (2011) LOVD v.2.0: the next generation in gene variant databases. Hum Mutat 32:557-563. https://doi.org/10.1002/humu.21438

30. Wildeman M, van Ophuizen E, den Dunnen JT, Taschner PEM (2008) Improving sequence variant descriptions in mutation databases and literature using the Mutalyzer sequence variation nomenclature checker. Hum Mutat 29:6-13. https://doi. org/10.1002/humu.20654

31. Hall TA (1999) BioEdit: a user-friendly biological sequence alignment editor and analysis program for Windows 95/98/NT. Nucleic Acids Symp Ser 41:95-98

32. Ellard S, De Franco E (2014) Next-generation sequencing for the diagnosis of monogenic diabetes and discovery of novel aetiologies. Front Diabetes 23:71-86. https://doi.org/10.1159/000362468 
33. Castellana S, Fusilli C, Mazza T (2016) Chap. 22 A broad overview of computational methods for predicting the pathophysiological effects of non-synonymous variants. Methods Mol Biol 1415:423-440. https://doi.org/10.1007/978-1-4939-3572-7

34. Ellard S, Bellanné-Chantelot C, Hattersley AT (2008) Best practice guidelines for the molecular genetic diagnosis of maturityonset diabetes of the young. Diabetologia 51:546-553. https:// doi.org/10.1007/s00125-008-0942-y

35. Murphy R, Ellard S, Hattersley AT (2008) Clinical implications of a molecular genetic classification of monogenic beta-cell diabetes. Nat Clin Pract Endocrinol Metab 4:200-213. https://doi. org/10.1038/ncpendmet0778

36. George DCP, Chakraborty C, Haneef SAS et al (2014) Evolutionand structure-based computational strategy reveals the impact of deleterious missense mutations on MODY 2 (maturity-onset diabetes of the young, type 2). Theranostics 4:366-385. https://doi. org/10.7150/thno.7473

37. Bowman P, Flanagan SE, Edghill EL et al (2012) Heterozygous ABCC8 mutations are a cause of MODY. Diabetologia 1:123127. https://doi.org/10.1007/s00125-011-2319-x

38. Ovsyannikova AK, Rymar OD, Shakhtshneider EV (2016) ABCC8-related maturity-onset diabetes of the young (MODY12): clinical features and treatment perspective. Diabetes Ther 7:591600. https://doi.org/10.1007/s13300-016-0192-9

39. Riveline J-P, Rousseau E, Reznik Y et al (2012) Clinical and metabolic features of adult-onset diabetes caused by ABCC 8 mutations. Diabetes Care 35:248-251. https://doi.org/10.2337/ dc11-1469

40. Klee P, Bellanné-chantelot C, Depret G et al (2012) A novel ABCC 8 mutation illustrates the variability of the diabetes phenotypes associated with a single mutation. Diabetes Metab 38:179-182. https://doi.org/10.1016/j.diabet.2011.12.001

41. Singh A, Satchell SC (2011) Microalbuminuria: causes and implications. Pediatr Nephrol 26:1957-1965. https://doi.org/10.1007/ s00467-011-1777-1

42. Pruhova S, Dusatkova P, Neumann D et al (2013) Two cases of diabetic ketoacidosis in HNF1A-MODY linked to severe dehydration: is it time to change the diagnostic criteria for MODY? Diabetes Care 36:2573-2574. https://doi.org/10.2337/dc13-0058

43. Egan AM, Cunningham A, Jafar-Mohammadi B, Dunne FP (2015) Diabetic ketoacidosis in the setting of HNF1A-maturity onset diabetes of the young. BMJ Case Rep 2015:bcr2014209163. https:// doi.org/10.1136/bcr-2014-209163

44. Fen S, Chi S, Sh C et al (2016) A preliminary study to evaluate the strategy of combining clinical criteria and next generation sequencing (NGS) for the identification of monogenic diabetes among multi-ethnic Asians. Diabetes Res Clin Pract 119:13-22. https://doi.org/10.1016/j.diabres.2016.06.008

45. Szopa M, Ludwig-Gałęzowska A, Radkowski P et al (2015) Genetic testing for monogenic diabetes using targeted nextgeneration sequencing in patients with maturity-onset diabetes of the young. Polish Arch Intern Med 125:845-851. https://doi. org/10.20452/pamw.3164

Publisher's Note Springer Nature remains neutral with regard to jurisdictional claims in published maps and institutional affiliations.

\section{Affiliations}

\section{Hamza Dallali ${ }^{1,2} \cdot$ Serena Pezzilli, ${ }^{3,4} \cdot$ Meriem Hechmi $^{1,2} \cdot$ Om Kalthoum Sallem ${ }^{5} \cdot$ Sahar Elouej $^{1,6} \cdot$ Haifa Jmel $^{1,7}$. Yosra Ben Halima ${ }^{1,8}$. Mariem Chargui ${ }^{1}$. Mariem Gharbi ${ }^{1}$. Luana Mercuri ${ }^{3}$. Federica Alberico ${ }^{3}$. Tommaso Mazza ${ }^{9}$. Afaf Bahlous $^{10}$. Melika Ben Ahmed ${ }^{11}$. Henda Jamoussi ${ }^{1,12}$. Abdelmajid Abid ${ }^{1,12}$. Vincenzo Trischitta ${ }^{3,4}$. Sonia Abdelhak ${ }^{1,8} \cdot$ Sabrina Prudente ${ }^{3} \cdot$ Rym Kefi $^{1,8}$}

1 Laboratory of Biomedical Genomics and Oncogenetics, Institut Pasteur de Tunis, BP 74, 13 Place Pasteur, 1002 Tunis, Tunisia

2 National Institute of Applied Sciences and Technology, University of Carthage, Tunis, Tunisia

3 Research Unit of Metabolic and Cardiovascular Diseases, Fondazione IRCCS Casa Sollievo della Sofferenza, San Giovanni Rotondo, Italy

4 Department of Experimental Medicine, Sapienza University, Rome, Italy

5 Fattouma Bourguiba University Hospital, Monastir, Tunisia

6 Faculty of Medicine La Timone, INSERM, GMGF, Aix Marseille University, 27 bd Jean Moulin, 13385 Marseille, France
7 Faculty of Sciences of Bizerte, University of Carthage, Tunis, Tunisia

8 University of Tunis El Manar, El Manar I, 2092 Tunis, Tunisia

9 Unit of Bioinformatics, IRCCS Casa Sollievo della Sofferenza, San Giovanni Rotondo, Italy

10 Central Laboratory of Medical Biology, Institut Pasteur de Tunis, BP 74, 13 Place Pasteur, 1002 Tunis, Tunisia

11 Laboratory of Transmission, Control and Immunobiology of Infections, Institut Pasteur de Tunis, BP 74, 13 Place Pasteur, 1002 Tunis, Tunisia

12 Research Unit on Obesity, National Institute of Nutrition and Food Technology, 11 rue Jebel Lakhdar, Bab Saadoun, 1007 Tunis, Tunisia 